\title{
SINTOMATOLOGÍA DE ANSIEDAD Y BURNOUT AL TÉRMINO DE LA LICENCIATURA DE MÉDICO
} CIRUJANO

\author{
ANXIETY AND BURNOUT SYMPTOMATOLOGY AT THE END OF MEDICINE CAREER \\ SINTOMATOLOGIA DE ANSIEDADE E BURNOUT NO FINAL DO CURSO DE MEDICINA \\ Ángel Alberto Puig-Lagunes ${ }^{1}$, Kevin Asiel Ricaño-Santos², Eliseo Quiroz Rojas², Román Darío González-Gutiérrez², \\ Ángel Puig-Nolasco ${ }^{3}$
}

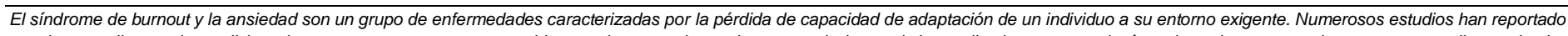

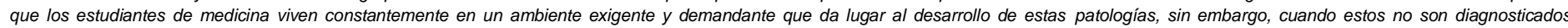

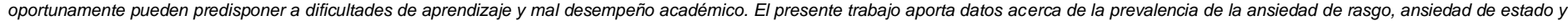

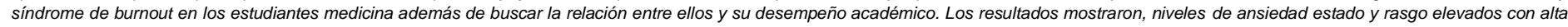
asociación entre ambos, la prevalencia de burnout fue baja y sin relación con el rendimiento académico.
\end{abstract}

\section{Resumen:}

La ansiedad y el síndrome de burnout son altamente frecuentes en los estudiantes de la licenciatura en Médico Cirujano, debido al demandante entorno y las exigencias académicas. El principal problema de estos, es que pocas veces son diagnosticados y se les da el seguimiento adecuado, favoreciendo a consecuencias como la dificultad para concentrarse y aprender lo cual repercute en el desempeño académico y ocasionando abandono escolar, además contribuye en el desarrollo de otras psicopatologías como la depresión. El presente trabajo aporta datos referentes a la prevalencia de las psicopatologías de ansiedad estado, rasgo y síndrome de burnout, así como su asociación con el rendimiento académico en estudiantes de medicina al término de la licenciatura. Además, refiere una gran alta asociación entre los dos tipos de ansiedad con el síndrome de burnout y como la ansiedad transitoria puede llegar a integrar una respuesta ansiosa como rasgo de la personalidad, es decir, que existe un riesgo elevado que aquellos estudiantes que presenten ansiedad estado y no se atiendan oportuna y debidamente desarrollen ansiedad rasgo.

${ }^{1}$ Doctorado en Investigaciones Cerebrales, perteneciente al Departamento de Ciencias básicas, Facultad de Medicina, Universidad Veracruzana, campus Minatitlán, Veracruz, México.

${ }^{2}$ Estudiante de la Facultad de Medicina, Universidad Veracruzana, campus Minatitlán, Veracruz, México.

${ }^{3}$ Doctorado en Neuroetología, encargado del Departamento de Sociomedicina, Facultad de Medicina, Universidad Veracruzana, campus Minatitlán, Veracruz, México. E mail de contacto: anpuig@uv.mx

Recibido: 2020-05-20 Aceptado: 2020-05-25

DOI: http://dx.doi.org/10.31053/1853.0605.v78.n1.28447 (c) (i) (8)

CUniversidad Nacional de Córdoba
Introducción: Las altas exigencias que presenta la carrera de medicina, aunado a las obligaciones laborales y personales de los estudiantes, los predispone a desarrollar ansiedad y síndrome de burnout (SB), lo cual puede influir en su rendimiento académico. El objetivo del presente estudio fue determinar la prevalencia y la sintomatología de ansiedad y burnout, así como identificar su impacto en el rendimiento académico de los estudiantes al término de la licenciatura en médico cirujano. Método: Se realizó un estudio transversal, observacional en estudiantes de medicina al término de la carrera, se utilizó el inventario de Ansiedad Rasgo y Estado, la escala Maslach burnout y el promedio de las calificaciones a través de la Secretaria Académica. Resultados: Se observó una prevalencia de ansiedad estado (AE) y rasgo (AR) de $40.6 \%$ y $31.2 \%$ respectivamente, ambas con proporciones similares entre nivel medio (75\%) y alto ( $25 \%$ ), y con asociación entre las mismas. La prevalencia del SB fue de $15.6 \%$, de los cuales 17.2 y $23.4 \%$ presentaron niveles altos de cansancio y de despersonalización respectivamente, además $18.8 \%$ mostró niveles bajos de realización personal. Conclusión: Al término de la licenciatura los estudiantes muestran sintomatología de $A R$ y $A E$ con alta asociación entre ambas, por lo que es importante resaltar que la ansiedad transitoria puede llegar a integrar una respuesta ansiosa como rasgo de su personalidad. La prevalencia de SB fue baja y sin relación con el rendimiento académico, la edad o el sexo.

Palabras clave: cansancio; despersonalización; realización personal; rendimiento académico; ansiedad rasgo; ansiedad estado; estudiante de medicina

\section{Abstract:}

Introduction: Due to the high demands of the medical career, coupled with students' work and personal obligations, they are a susceptible population to develop anxiety and burnout syndrome (BS) which can influence their academic performance. The aim of the present study was to determine the prevalence and symptomatology of anxiety and burnout, as well as to identify their impact on the academic performance of students at the end of their medical degree. Method: A cross-sectional, observational study was conducted on medical students at the end of their degree, using the Trait and State Anxiety inventory, the Maslach burnout scale, and the grade point average through the Academic Secretary. Results: A prevalence of state anxiety (SA) of $40.6 \%$ and trait anxiety (TA) of $31.2 \%$ was observed, both with similar proportions between a medium and high level (75-25\%), with an association between them. The prevalence of BS was $15.6 \%$, of which $17.2 \%$ and $23.4 \%$ respectively showed high levels of fatigue and depersonalization, moreover, 18. $8 \%$ showed low levels of personal fulfillment. Conclusion: At the end of the career, students show symptoms of SA and TA with a high association between them. Therefore, it is important to emphasize that transitory anxiety can become part of an anxious response as a personality trait. The prevalence of BS was low and not related to academic performance, age, or sex.

Keywords: fatigue; depersonalization; personal fulfillment; academic performance; trait anxiety; state anxiety; medical student

\section{Resumo}

Introdução: Devido às elevadas demandas presentes nos cursos de medicina, associadas as múltiplas obrigações escolares e pessoais, os estudantes de medicina estão suscetíveis ao desenvolvimento da ansiedade e síndrome de burnout (BS), o que pode influenciar em seu desempenho acadêmico. Desta forma, o objetivo do presente estudo foi determinar a prevalência e os níveis de ansiedade e sintomatologia de burnout, bem como avaliar seu impacto no desempenho acadêmico dos estudantes no final do curso de medicina. Método: Estudo observacional, transversal, realizado em estudantes de medicina no final do curso, utilizando o Inventário de Traço e Ansiedade Estatal, a escala Maslach burnout e as médias do desempenho acadêmico obtidas na cordinaçao acadêmica. Resultados: Observou-se prevalência de ansiedade de estado de 40,6 \% e de característica de 31,2\%, ambas em proporções semelhantes entre nível médio e alto (75-25\%, respectivamente), as quais demonstram uma alta correlação. A prevalência da Síndrome de burnout foi de $15,6 \%$, dos quais 17,2 e $23,4 \%$ apresentaram altos níveis de fadiga e despersonalização, respectivamente, além disso, 18,8 \% apresentaram baixos níveis de realização pessoal. Conclusão: No final do curso, os alunos apresentaram sintomas de traço e estado de ansiedade com elevada correlação entre ambos, por isso é importante enfatizar que a ansiedade transitória pode integrar uma resposta ansiosa como traço de personalidade. A prevalência da síndrome de burnout foi baixa, sem relação com o desempenho acadêmico, idade ou sexo.

Palavras-chaves: fadiga; despersonalização; realização pessoal; desempenho académico; traços de traço, estado de estatal; estudante de medicina. 
Introducción

El estudiante de medicina está sometido a un ambiente de trabajo exigente, demandante y socialmente limitado a su entorno, que en ocasiones puede ser hostil y promover mayor vulnerabilidad a desarrollar trastornos psicológicos como la ansiedad y depresión ${ }^{(1-3)}$.

Normalmente, la ansiedad se presenta como una condición biológica de respuesta, pero cuando excede la capacidad de adaptación al ambiente se torna patológica y es acompañada de una gama de alteraciones psicológicas y psicosomáticas, entendida como ansiedad de rasgo (AR) y estado $(A E)^{(3-5)}$, siendo la primera, parte de la personalidad del individuo por lo que es permanente; y la segunda, hace referencia al estado emocional en determinado momento, por lo que es fluctuante ${ }^{(3-4)}$. De acuerdo con la Organización Mundial de la Salud, $15 \%$ de la población mundial puede tener ansiedad y $20 \%$ puede desarrollarla ${ }^{(6)}$. Entre los estudiantes de medicina, la prevalencia de AR y AE oscila entre $19-43 \%$ para ambos $\operatorname{casos}^{(5,7)}$.

Por otra parte, cuando los estudiantes no logran adaptarse a los eventos de ansiedad se pueden desencadenar sensaciones de agotamiento, con actitud de desvalorización, perdida de interés o deshumanización, e incrementar las dudas de su valor y realización personal, lo que en conjunto son indicadores del síndrome de burnout $(\mathrm{SB})^{(8-9)}$. Mundialmente alrededor de $4.2 \%$ de la población podría tener $\mathrm{SB}^{(10)}$, y entre los estudiantes de medicina esta prevalencia asciende de 37 a $75 \%{ }^{(11-12)}$.

Tanto la ansiedad como el SB pueden llegar afectar la salud y el aprendizaje, convirtiéndose en factores de riesgo para bajo rendimiento académico, lo que podría repercutir en el desarrollo de habilidades clínicas y la toma de decisiones en su futuro profesional ${ }^{(7,13)}$. Por tales motivos, el objetivo del presente estudio es determinar la prevalencia y los niveles de sintomatología de ansiedad y de SB, así como valorar su impacto en el rendimiento académico de los estudiantes al término de la licenciatura en médico cirujano.

\section{Métodos}

Se realizó un estudio transversal, observacional y descriptivo en los estudiantes de la generación 2013-2018 al término de la licenciatura de médico cirujano, garantizándoles la confidencialidad de los datos proporcionados por medio de un consentimiento informado, de acuerdo con la Declaración de Helsinki. Se tomaron como criterios de inclusión a todos los estudiantes inscritos en el periodo mencionado y que hubiesen acreditado todas las asignaturas para concluir la licenciatura. No se incluyeron aquellos estudiantes que no aceptaron participar y no completaron en su totalidad los cuestionarios. El diseño del estudio se revisó y aprobó por el Comité de Ética Institucional (Folio CIE0022019).

\section{Instrumentos}

Para determinar la sintomatología de ansiedad se utilizó el inventario de AR y AE (IDARE) que consta de cuarenta ítems, los primeros veinte miden la $A E$ (como se siente en esos momentos) y los veinte subsecuentes la AR (característica de la personalidad permanente); los sujetos seleccionaron una de las cuatro categorías que más se apegaba a como se sentían de acuerdo con el contenido de cada ítem: no (1), un poco (2), bastante (3) y mucho (4). Obteniendo una puntuación de $A E$ y otra de $A R$ con valores que oscilan entre 20 y 80 puntos. El resultado clasifica en distintos niveles de ansiedad cada escala siendo: muy bajo (20-31) y bajo (32-43) ambas categorías clasificadas sin ansiedad; nivel medio (44-55), alto (56-67) y muy alto $(68-80)$ consideradas con ansiedad ${ }^{(3-4)}$.

EI SB se evaluó con el Maslach Burnout Inventory (MBI), que permite conocer su presencia por medio de tres subescalas: a) Desgaste emocional, consta de 9 ítems que valoran la vivencia de estar exhausto emocionalmente por las demandas laborales; b) Despersonalización, conformada por 5 ítems los cuales valoran el grado de reconocimiento de actitudes de frialdad y distanciamiento; c) Realización personal compuesta de 8 ítems, evalúan los sentimientos de autoeficacia y realización personal en el trabajo. Cada ítem contiene las respuestas: nunca (0), pocas veces al año o menos (1), una vez al mes o menos (2), unas pocas veces al mes o menos (3), una vez a la semana (4), pocas veces a la semana (5) y todos los días (6). El diagnóstico del SB se obtuvo basándose en las altas puntuaciones en las subescalas de desgaste emocional (>26), despersonalización $(>9)$ y la asociación de puntuaciones bajas en la subescala de realización personal $(<34)^{(9,14)}$.

Finalmente, el promedio de las calificaciones se obtuvo del cárdex de la Secretaria Académica y se clasificaron en: bajo $<7.9$, regular de 8 a 8.9 y alto $>9$.

\section{Análisis Estadístico}

Los análisis univariados se calcularon por medio de frecuencias, porcentajes y medidas de tendencia central y dispersión. La asociación estadística entre la ansiedad estado-rasgo y el SB con las variables sociodemográficas se realizaron mediante análisis bivariados por medio de Chi cuadrada $\left(\mathrm{X}^{2}\right)$. Se realizaron análisis de varianza (ANOVA) para comparar entre promedios y grupos etarios. Se utilizó un nivel de significancia de $<0.05$. Los datos fueron analizados con el software IBM SPSS Statistics versión 21 para MacOS.

\section{Resultados}

De un total de 72 estudiantes de la generación 2013-2018, aceptaron participar y respondieron los cuestionarios en su totalidad $64(88.8 \%)$, de los cuales 33 eran mujeres ( $51.6 \%$ ) y 31 hombres (48.4\%), en una edad promedio de 23.3 años (rango 22 a 27 años) y un promedio de calificaciones de 8.54 (rango 7.60 a 9.57).

Se encontró una prevalencia de AE en $40.6 \%$ de los estudiantes, de los cuales $73.1 \%$ mostraron nivel medio y $26.9 \%$ alto. Referente al sexo, se observó en $42.4 \%$ de las mujeres y $38.7 \%$ de los hombres (Tabla 1), no se evidenció diferencias significativas entre sexos $(p=0.960)$.

Se observó qué $62.5 \%$ de los estudiantes con promedio bajo presentaron sintomatología de $\mathrm{AE}$, mientras que en los estudiantes con promedio regular y alto fue de $37 \%$ y $40 \%$ respectivamente. Con respecto a la edad, los estudiantes mayores de 25 años presentaron $62.5 \%$ de $A E$, seguidos de los de $21-22$ años con $54.5 \%$, y $33.3 \%$ en los de 23-24 años. Se encontraron diferencias significativas entre los grupos etarios $(\mathrm{p}=0.0494)$, sin embargo, no se observó asociación entre la $A E$ y la edad $(p=0.165)$.

Tabla 1

\begin{tabular}{|c|c|c|c|c|c|c|c|c|c|}
\hline & \multicolumn{9}{|c|}{ Ansiedad Estado } \\
\hline & \multirow{2}{*}{$\begin{array}{l}\text { Total } \\
n=64\end{array}$} & \multicolumn{3}{|c|}{ Promedio } & \multicolumn{2}{|c|}{ Sexo } & \multicolumn{3}{|c|}{ Edades } \\
\hline & & $\begin{array}{c}\text { Bajo } \\
n=8\end{array}$ & $\begin{array}{c}\text { Regular } \\
\mathrm{n}=46\end{array}$ & $\begin{array}{c}\text { Alto } \\
\mathrm{n}=10\end{array}$ & $\begin{array}{c}\stackrel{9}{+} \\
\mathrm{n}=33\end{array}$ & $\underset{n=31}{\hat{O}}$ & $\begin{array}{c}21- \\
22 \\
n=11\end{array}$ & $\begin{array}{c}23- \\
24 \\
n=45\end{array}$ & $\begin{array}{l}>25 \\
\mathrm{n}=8\end{array}$ \\
\hline Síntomas & 40.6 & 62.5 & 37.0 & 40.0 & 42.4 & 38.7 & 54.5 & 33.3 & $62.5^{*}$ \\
\hline Medio & 73.1 & 80.0 & 76.4 & 50.0 & 71.4 & 75 & 66.6 & 73.3 & 80 \\
\hline Alta & 26.9 & 20.0 & 23.4 & 50.0 & 28.6 & 25 & 33.3 & 26.6 & 20 \\
\hline
\end{tabular}

* $p<0,05$, Valores expresados en porcentajes

Tabla 1.- Prevalencia de ansiedad estado en los estudiantes de medicina campus Minatitlán.

Se observó una prevalencia de AR en $31.2 \%$ estudiantes, de los cuales $75 \%$ mostraron nivel medio y $25 \%$ alto. De acuerdo con el sexo,

39.3. \% de las mujeres y $22.2 \%$ de los hombres presentan AR, sin mostrar diferencias entre sexos $(p=0.961)$. Referente a las calificaciones, se observó mayor prevalencia en los estudiantes con promedio bajo (37.5\%), seguido de los de promedio regular $(30.4 \%)$ y alto (30\%) (Tabla 2), no se encontró diferencias entre las calificaciones $(\mathrm{p}=0.4322)$.

De acuerdo con la edad, los mayores de 25 años y los de 21-22 años presentaron prevalencias similares (37.5 y $36.3 \%$ respectivamente), y $29.5 \%$, en los de $23-24$ años, de los cuales no mostraron diferencias estadísticas entre los grupos etarios ( $\mathrm{p}=0.4542)$. 


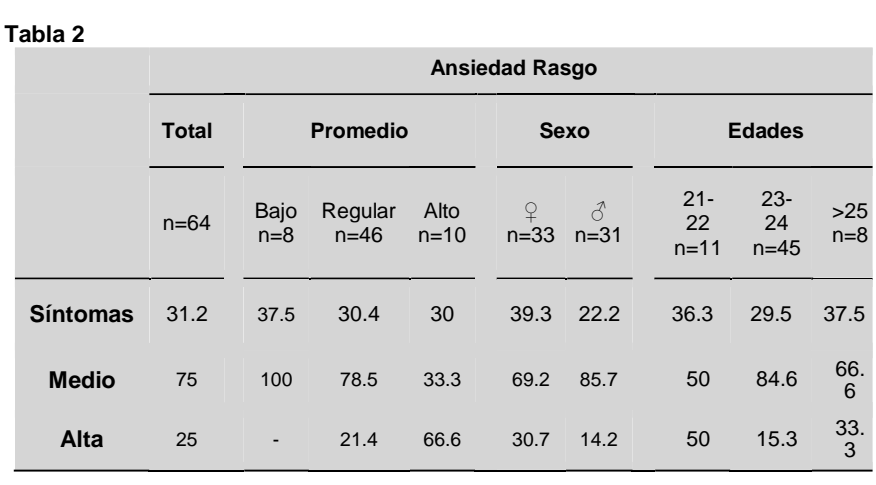

Tabla 2.- Prevalencia de ansiedad rasgo en los estudiantes de medicina campus Minatitlán.

Se encontró asociación entre la AE y AR ( $\mathrm{p}<0.000)$, además se observó concordancia entre los niveles de ansiedad, debido a que el $78.5 \%$ de los estudiantes con nivel medio de AE mostraron el mismo nivel de AR, de forma similar el $80 \%$ de los estudiantes con nivel alto de $A E$ presentaron AR (Tabla 3).

Tabla 3.

\begin{tabular}{l|c|ccccc} 
& \multicolumn{7}{|c}{ Ansiedad Estado } \\
\cline { 2 - 6 } & Niveles & $\begin{array}{c}\text { Muy } \\
\text { bajo }\end{array}$ & Bajo & Medio & Alto & Total \\
Muy bajo & 10 & 4 & 0 & 0 & 14 \\
$\begin{array}{l}\text { Ansiedad } \\
\text { Rasgo }\end{array}$ & Bajo & 7 & 16 & 1 & 0 & 24 \\
Medio & 2 & 5 & 11 & 1 & 19 \\
Alto & 0 & 0 & 3 & 4 & 7 \\
Total & 19 & 25 & 15 & 5 & 64
\end{tabular}

Tabla 3.- Relación entre los niveles de ansiedad-estado y ansiedadrasgo en los estudiantes de medicina campus Minatitlán.

La prevalencia de burnout fue $15.6 \%$, de los cuales $40 \%$ eran mujeres y $60 \%$ hombres. Se encontró que 17.2 y $23.4 \%$ de los estudiantes presentaron niveles altos de cansancio y de despersonalización respectivamente, además de un $18.8 \%$ de niveles bajos de realización personal (Tabla 4), sin diferencias respecto al sexo $(p=0.426)$. Por otro lado, $15.7 \%$ de la población que no fue diagnosticada con SB se encuentra en riesgo de padecerlo.

Tabla 4.

\begin{tabular}{c|ccc} 
& \multicolumn{3}{|c}{ Escalas MBI } \\
\cline { 2 - 4 } Sub-escalas & Bajo & Moderado & Alto \\
Cansancio Emocional & 64 & 18.7 & 17.2 \\
Despersonalización & 59.3 & 17.2 & 23.4 \\
Realización personal & 18.8 & 29.7 & 51.5
\end{tabular}

Valores representados en porcentajes

Tabla 4.- Distribución de las subescalas del síndrome de burnout en los estudiantes de medicina campus Minatitlán.

Se encontraron diferencias significativas respecto a la edad $(p<0.000)$, debido a que casi la totalidad de los estudiantes con SB tienen entre 21 y 22 años. Se observó SB en $60 \%$ de los estudiantes con promedio regular, $30 \%$ con promedio alto y $10 \%$ con promedio bajo, sin diferencias significativas entre promedios $(p=0.65)$.
Se encontró asociación entre SB y la severidad de $A E(p<0.000)$ y $A R$ $(p<0.000)$. Se evidenció que existe una mayor proporción de estudiantes con SB y AE-AR que, de estudiantes sin burnout y ansiedad, ya que el $80 \%$ de los estudiantes con SB presentó AE y el $70 \%$ presentó AR. Es importante mencionar que el $67 \%$ de los estudiantes que presentaron $A E$ también mostró $A R$, es decir, solo en el $23 \%$ restantes no se encontraba implícito la AR.

\section{Discusión}

Los resultados muestran que al término de la licenciatura de médico cirujano los estudiantes presentan una alta prevalencia de sintomatología de ansiedad, ya que uno de cada tres estudiantes muestra síntomas de AE y AR, con asociación estrecha entre ambas y con el SB, pero sin relación con el rendimiento académico; la prevalencia de burnout es baja y se observó en mayor predominancia en los egresados de menor edad.

La prevalencia de AR y AE observada supera a los datos obtenidos en otras universidades Latinoamericanas de medicina ${ }^{(2,15-16)}$. Las diferencias pudieran deberse a diversos factores como el instrumento de aplicación ${ }^{(2,17)}$, y las poblaciones en las que se aplicaron, debido a que algunos estudios incluyen estudiantes desde el primer hasta el último año ${ }^{(4,13,18)}$. Se observó que los estudiantes con calificaciones bajas presentaban una prevalencia mayor en $A E$ y $A R$, como lo han mencionado otros estudios ${ }^{(17)}$. Se observó mayor frecuencia de $A E-A R$ en los estudiantes mayores de 25 años, posiblemente asociado a las responsabilidades fuera del ámbito académico, como tener familia o trabajar $^{(17-18)}$.

Se observó asociación entre ambos tipos de ansiedad, debido a que tres de cada cuatro estudiantes con AE también presentan AR, por lo que se deben implementar estrategias de monitoreo y diagnóstico para evitar que aquellos con ansiedad transitoria puedan desarrollar una respuesta ansiosa como rasgo de su personalidad ${ }^{(1,11)}$. Además, deben fomentarse la práctica de actividades extracurriculares para evitar la progresión de $A E$ a $A R$, donde es más frecuente que continúe su degeneración y puedan aparecer otras psicopatologías como depresión ${ }^{(19-21)}$. Desempeñar actividades extracurriculares como leer, realizar actividades físicas, sociales, tocar algún instrumento musical disminuyen esta prevalencia hasta en $20 \%{ }^{(16)}$.

Se encontró una prevalencia baja de burnout en comparación con otros reportes que rondan entre $24-70 \%{ }^{(22-24)}$. Estas diferencias pueden deberse a factores socioculturales, las poblaciones de estudio y la variabilidad de modelos educativos ${ }^{(12,22,25)}$. Aunque se tiene la firmeza que el SB se relaciona con el bajo rendimiento académico ${ }^{(5,20)}$, en este estudio no se encontró relación, lo se ha reportado por otros autores ${ }^{(24)}$, sin embargo, se observó una mayor prevalencia en aquellos con promedio regular-alto, posiblemente porque estos estudiantes se estresan por mantener un mayor promedio; también hay que tomar en cuenta que la población estudiada presenta una baja proporción de estudiantes con calificaciones bajas, lo cual influye en dicha asociación. Por otro lado, se observó mayor prevalencia de burnout entre los estudiantes de 20-21 años lo cual ya ha sido reportado ${ }^{(26)}$, quizá porque en esta edad se carece de estrategias adecuadas para afrontar los problemas que se generan en las prácticas clínicas, además de la falta de reconocimiento como profesionales.

Por todo lo anterior, es indispensable establecer programas de monitoreo a lo largo de la carrera para la detección, prevención y abordaje de psicopatologías, aunado a la implementación de estrategias para contrarrestarlas como las actividades extracurriculares, además es necesario tomar en cuenta otras variables que pueden influir como: las sociodemográficas, calidad de sueño, consumo de sustancias lícitas e ilícitas, bebidas energéticas, alimentación, entre otras.

\section{Conclusión}

Al término de la licenciatura de médico cirujano los estudiantes muestran sintomatología de ansiedad de rasgo y estado con alta asociación entre ambas, resaltando que la ansiedad transitoria puede llegar a integrar una respuesta ansiosa como rasgo de su personalidad. Por otra parte, se detectó síndrome de burnout, con asociación a la ansiedad, pero ambas sin influencia con el rendimiento académico. 


\section{Limitaciones de responsabilidad}

La responsabilidad del trabajo es exclusivamente de los autores.

\section{Conflictos de interés}

Ninguno

\section{Fuentes de apoyo}

No posee

\section{Originalidad del trabajo}

Este artículo es original y no ha sido enviado para su publicación a otro medio de difusión científica en forma completa ni parcialmente.

\section{Sesión de derechos}

Los participantes de este trabajo ceden el derecho de autor a la Universidad Nacional de Córdoba para publicar en la Revista de la Facultad de Ciencias Médicas y realizar las traducciones necesarias al idioma inglés.

\section{Participación de los autores}

Todos los autores han participado en la concepción del diseño, recolección de la información y elaboración del manuscrito, haciéndose públicamente responsables de su contenido y aprobando su versión final.

\section{Bibliografía}

1.- Gutiérrez Rodas JA, Montoya Vélez LP, Toro Isaza BE, Briñón Zapata MA, Rosas Restrepo E, Salazar Quintero LE. «Depresión en estudiantes universitarios y su asociación con el estrés académico». CES Medicina, 2010;24(1): 7-17.

2.- Jafari N, Loghmani A, Montazeri A. «Mental Health of Medical Students in Different Levels of Training». International Journal of Preventive Medicine, 2012;3(1):S107-112.

3.- Castillo Pimienta C, Chacón de la Cruz T, Díaz-Véliz G. "Ansiedad y fuentes de estrés académico en estudiantes de carreras de la salud". Investigación en Educación Médica, 2016;5(20):230-37.

4.- Reyes Carmona C, Monterrosas Rojas AM, Navarrete Martínez A, Acosta Martínez EP, Torruco García U. «Ansiedad de los estudiantes de una facultad de medicina mexicana, antes de iniciar el internado". Investigación en Educación Médica, 2017;6(21):42-46.

5.- García Gascón A, Querts Méndez O, Hierrezuelo Izquierdo EJ, Quesada Vidal S. "Algunos aspectos psicosociales del insomnio en estudiantes de primer año de medicina». MEDISAN, 2015;19(1):56-60. 6.- Hernández RA, Flores JFL, Flores RCF. «Prevalencia de la ansiedad y depresión de médicos residentes de especialidades médicas». Evid Med Invest Salud, 2011;6(8)28-33.

7.- Aguilera MI, Ajpop F, Aqueche G, Bámaca E, Bolaños D, Estrada $H$, et al. «Niveles de Ansiedad de Médicos Residentes». Rev Guatem Cir, 2015;21:(7):22-28.

8. - Segura O. "Agotamiento profesional: concepciones e implicaciones en la salud pública» Biomédica, 2014;34(4):535-45.

9.- Ramírez MR. «Prevalencia del síndrome de burnout y la asociación con variables sociodemográficas y laborales en una provincia de Ecuador». Int J Dev Educ Psychol Rev INFAD Psicol, 2017;4(1):241251.

10.- Rosales Ricardo Yury, Rosales Paneque FR. «Burnout estudiantil universitario: Conceptualización y estudio». Salud mental, 2013;36(4):337-45.

11.- Jawad F, Saadeddin Z, Al Tabosh H, Aridi H, El Mouhayyar C, Karim Koleilat M, Chaaya M, El Asmar K. «Extracurricular activities associated with stress and burnout in preclinical medical students". Journal of Epidemiology and Global Health, 2016;6(3):177-85.

12.- Galán F, Sanmartín A, Polo J, Giner L. «Burnout Risk in Medical Students in Spain Using the Maslach Burnout Inventory-Student Survey». International Archives of Occupational and Environmental Health, 2011;84(4):453-59.
13.- Brenneisen Mayer F, Souza Santos IP, Silveira PS, Itaqui Lopes MH, Navarro Dias de Souza AR, Paes Campos E, et al. "Factors associated to depression and anxiety in medical students: a multicenter study». BMC Medical Education, 2016;16(1):282.

14.- Hernández Vargas Cl, Llorens Gumbau S, Rodríguez-Sánchez A. "Burnout en personal sanitario: validación de la escala MBI en México». Fórum de recerca, 2011;(16):837-46.

15.- Galván-Molina JF, Jiménez-Capdeville ME, Hernández-Mata JM, Arellano-Cano JR. "Sistema de tamizaje de psicopatología en estudiantes de Medicina”. Gaceta Médica de México, 2017;153(1):7587.

16.- Lemos M. «Estrés y Salud Mental en Estudiantes de Medicina: Relación con Afrontamiento y Actividades Extracurriculares». Archivos de Medicina, 2018;14(2):8.

17.- Serrano Barquín C, Rojas García A, Ruggero C. "Depresión, ansiedad y rendimiento académico en estudiantes universitarios". Revista Intercontinental de Psicología y Educación, 2013;15(1):47-60.

18.- Guerrero López JB, Heinze Martin G, Ortiz de León S, Cortés Morelos J, Barragán Pérez V. «Factores que predicen depresión en estudiantes de medicina». Gaceta Médica de México, 2013;149:598604.

19.- Vilchez-Cornejo J, Huamán-Gutiérrez R, Arce-Villalobos L, MoránMariños C, Mihashiro-Maguiña K, Melo-Mallma $N$, EyzaguirreVillagarcia J, Rojas-Valle C. "Síndrome de burnout en estudiantes de medicina: frecuencia, características y factores asociados». Acta Médica Peruana, 2016;33(4):282-88.

20.- Alfaro Toloza P, Olmos de Aguilera R, Fuentealba M, Céspedes González $E$. "Síndrome de burnout y factores asociados en estudiantes de una escuela de medicina de chile». Ciencia e Investigación Medico Estudiantil Latinoamericana, 2013;18(2):23-26.

21.- Caballero-Dominguéz CC, González Gutiérrez O, Palacio Sañudo JE. «Relación del burnout y el engagement con depresión, ansiedad y rendimiento académico en estudiantes universitarios". Revista Científica Salud Uninorte, 2015;32(2):254-267.

22.- Asencio-López L, Almaraz-Celis GD, Carrillo Maciel V, Huerta Valenzuela P, Silva Goytia L, Muñoz Torres M, Monroy Caballero F, et al. "Síndrome de burnout en estudiantes de primero a sexto año de medicina en una universidad privada del norte de México: estudio descriptivo transversal». Medwave, 2016;16(3)e6432.

23.- Nakandakari Mayron D, De la Rosa DN, Failoc-Rojas VE, Huahuachampi KB, Nieto WC, Gutiérrez Al, Ruiz FN, et al. "Síndrome de burnout en estudiantes de medicina pertenecientes a sociedades científicas peruanas: Un estudio multicéntrico." Revista de NeuroPsiquiatria, 2016;78(4):203-210.

24.- Costa EF, Santos SA, Sanntos AT, Melo EV, Andrade TM. "Burnout Syndrome and Associated Factors among Medical Students: A Cross-Sectional Study». Clinics, 2012;67,(6):573-80.

25.- Serrano FT, Salguero Sánchez J, Ayala Fernandez, J, García MF, Meza JC, y Mejía Alvarez CR. "Síndrome de burnout en estudiantes de 6 facultades de medicina de Colombia, 2016-1: estudio multicéntrico". Ciencia e Investigación Medico Estudiantil Latinoamericana, 2016;21(2):29-34.

26.- Gilson de Cavalcante A, Ribeiro de Souza H, César de Almeida P, de Cavalcante Almeida B, Holanda Almeida G. "The Prevalence of burnout syndrome in Medical Students». Archives of Clinical Psychiatry, 2016;43(1):6-10. 